\title{
Improving the Affordability of Prescription Medications for People with Chronic Respiratory Disease: An Official American Thoracic Society Policy Statement
}

Minal R. Patel, PhD, MPH${ }^{1}$; Valerie G. Press, MD, MPH'; Lynn B. Gerald, PhD, MSPH ${ }^{3,4}, \mathrm{MSPH}^{2}$ Teresa Barnes, BA ${ }^{5}$; Kathryn Blake, Pharm.D.6; Lee K. Brown, MD7; Richard W. Costello, MD ${ }^{8}$; Courtney Crim, MD ${ }^{9-11}$; Mark Forshag, MD, MHA ${ }^{10-11}$; Andrea Gershon, MD, MSc (ORCID: 0000-00020246-594X), FRCPC ${ }^{12}$; Christopher H. Goss, MD, MS, FCCP ${ }^{13}$; MeiLan K. Han, MD, MS ${ }^{14}$; Todd A. Lee, PhD, PharmD ${ }^{15}$; Stuart Sweet, MD, PhD ${ }^{16}$ (ORCID: 0000-0002-0638-2586) ; Joe K. Gerald, MD, $\mathrm{PhD}^{4,17}$ on behalf of the Assembly on Behavioral Sciences and Health Services Research

${ }^{1}$ Department of Health Behavior \& Health Education, University of Michigan School of Public Health; ${ }^{2}$ Departments of Medicine and Pediatrics, University of Chicago; ${ }^{3}$ Department of Health Promotion Sciences, The Mel and Enid Zuckerman College of Public Health; ${ }^{4}$ The Asthma and Airway Disease Research Center; ${ }^{5}$ American Thoracic Society Patient Advisory Council; ${ }^{6}$ Center for Pharmacogenomics and Translational Research, Nemours Children's Specialty Care; ${ }^{7}$ Division of Pulmonary, Critical Care, and Sleep Medicine, University of New Mexico School of Medicine;

${ }^{8}$ Department of Medicine, Royal College of Surgeons in Ireland; ${ }^{9}$ Division of Pulmonary \& Critical Care Medicine, University of North Carolina-Chapel Hill; ${ }^{10} \mathrm{GlaxoSmithKline,} \mathrm{Research} \mathrm{Triangle} \mathrm{Park,} \mathrm{NC;}$

${ }^{11}$ American Thoracic Society Drug Device Discovery and Development Committee; ${ }^{12}$ Sunnybrook Health Sciences Centre and Institute for Clinical Evaluative Sciences, University of Toronto; ${ }^{13}$ Division of Pulmonary, Critical Care and Sleep Medicine, University of Washington; ${ }^{14}$ Division of Pulmonary and Critical Care Medicine, University of Michigan; ${ }^{15}$ Pharmacy systems, Outcomes, and Policy, College of Pharmacy, University of Illinois at Chicago; ${ }^{16}$ Division of Allergy, Immunology and Pulmonary Medicine, Washington University School of Medicine; ${ }^{17}$ Department of Community Environment and Policy, The Mel and Enid Zuckerman College of Public Health

Funding: This work was supported by the American Thoracic Society

\section{Corresponding Authors}

Minal R. Patel, PhD, MPH

Department of Health Behavior \& Health Education

University of Michigan School of Public Health

1415 Washington Heights, SPH 1, Room 3810

Ann Arbor, Ml 48109-2029

minalrp@umich.edu 
36 Joe K. Gerald, MD, PhD

37 Department of Community Environment and Policy

38 Mel and Enid Zuckerman College of Public Health

39 The Asthma and Airways Disease Research Center

40 The University of Arizona

41 geraldj@email.arizona.edu

42

43 Word count

44 Abstract: 245

45 Manuscript: 2999

46

47

48 


\section{ABSTRACT}

Background: Mounting evidence indicates that out-of-pocket costs for prescription medications, particularly among low- and middle-income patients with chronic diseases, are imposing financial burden, reducing medication adherence, and worsening health outcomes. This problem is exacerbated by a paucity of generic alternatives for prevalent lung diseases such as asthma and COPD as well as high cost medicines for rare diseases such as cystic fibrosis. Affordability and access challenges are especially salient in the U.S, as citizens of many other countries pay lower prices for and have greater access to prescription medications.

Methods: The American Thoracic Society convened a multidisciplinary committee comprising experts in health policy, pharmacoeconomics, behavioral sciences and clinical care, along with individuals providing industry and patient perspectives. The report and its recommendation were iteratively developed over a year of in-person, telephonic, and electronic deliberation.

Results: The committee unanimously recommended the establishment of a publicly funded, politically independent, impartial entity to systematically draft evidence-based pharmaceutical policy recommendations. The goal of this entity would be to generate evidence and action steps to ensure people have equitable and affordable access to prescription medications, to maximize the value of public and private pharmaceutical expenditures on health, to support novel drug development within a market-based economy, and preserve clinician and patient choice regarding personalized treatment. An immediate priority is to examine the evidence and make recommendations regarding the need to have essential medicines with established clinical benefit from each drug class in all Tier 1 formularies, and propose recommendations to reduce barriers to timely generic drug availability.

Conclusions: By making explicit, evidence-based recommendations, the entity can support the establishment of coherent national policies that expand access to affordable medications, improve the health of patients with chronic disease, and optimize the use of public and private resources. 


\section{TABLE OF CONTENTS}

75 Executive Summary

76 Background

77 Description of Committee Composition and Activity

78 The Pharmaceutical Supply Chain in the United States

79 Pharmaceutical Supply Chain in the International Context

80 Recommendation

81 Conclusion

82

83

84

85 


\section{EXECUTIVE SUMMARY}

Rising healthcare costs are leading to higher out-of-pocket (OOP) expenses for patients such that one-quarter of families report health spending imposes a significant financial burden. This burden is especially high among individuals with low and middle incomes who have chronic respiratory conditions and is magnified by the limited availability of generic medicines within many drug classes. Even when generics are available, their prices are unexpectedly high due to lack of competition. Rising medication costs increase stress and fear while cost-related non-adherence worsens health status and leads to unnecessary hospitalizations. Other economically developed countries have taken greater steps than the U.S. to establish policies to protect patients from unmanageable prescription medication expenses. There is a pressing need in the U.S. to establish more consistent and rational pharmaceutical policies at the national level.

The current market for prescription medications is complex and contains numerous government and private entities that regulate, develop, distribute, dispense and purchase prescription medications. Recommendations addressing affordability must acknowledge the diverse roles these entities play in making medications available to patients. The National Academies of Science, Engineering and Medicine and others recommend important reforms based on careful and objective examination of available evidence. Given the difficulty of crafting and implementing evidence-based policies in a complex and dynamic pharmaceutical supply chain, a system is needed to monitor long-term outcomes and adapt to changing circumstances.

The following policy recommendations are designed to address the long-term challenges facing the nation as a whole, in order to better serve all patients in need of prescription medications, not just those with respiratory disease:

- The U.S. government should establish and financially support a politically independent, impartial expert advisory committee tasked with making critical, evidence-based recommendations on pharmaceutical policy. Their overarching goals would be to ensure affordable access to life- 
111 preserving medications, while retaining consumer choice, promoting future innovation and 112 respecting our market-based economy.

114 - An immediate priority of the aforementioned committee is to examine the evidence and make 115 strong recommendations to have essential medicines with established clinical benefit from each 116 drug class in all Tier 1 formularies, and reduce barriers to timely generic drug availability. 


\section{BACKGROUND}

Prescription medication spending has garnered notable attention in the U.S. because rising expenditures are straining government and patient finances. Demand for prescription medications is high given $50 \%$ of adults have at least one chronic condition and $25 \%$ have two or more (1). Sixty percent of the population and $90 \%$ of Medicare beneficiaries have taken at least one prescription medication in the past month (2). While prescription medication spending accounts for $22 \%$ of all expenditures devoted to treating individual medical conditions, it accounts for $28 \%$ among those with chronic respiratory conditions and 58\% among those with asthma, a highly prevalent disease (3-4). Prescription medications are critical to the effective management of respiratory disease, which is reflected in increased spending. However, rising healthcare costs are passed on to patients through higher out-of-pocket (OOP) expenses resulting in one-quarter of families reporting a significant financial burden (5). This burden is greatest among individuals with low and middle incomes who have a chronic condition, with OOP spending doubling between zero and one condition, and between two and multiple conditions (6).

Higher OOP medication costs imposes a financial burden on families that is known to increase stress and fear, and to induce cost-related non-adherence--- an adaption that affects nearly one-third of adults with chronic conditions, worsens health status, and leads to unnecessary hospitalizations (7-9). Among economically developed countries, cost-related non-adherence is highest in the U.S (10). Costsaving tactics includes taking smaller doses, skipping doses, delaying refills and borrowing medicines from others (11). Transferring medication costs from third-party payers to patients may burden patients without yielding corresponding savings if non-adherence leads to increased health care utilization in other service settings (e.g., hospital admissions).

The high cost of prescription medications coincides with increases in all forms of health care spending (12). While health spending is highest in the U.S., all economically developed nations struggle to ensure their health expenditures are fiscally sustainable (12). Other economically developed countries have taken greater steps than the U.S. to use national policies to curb health spending and 
144 protect patients from OOP expenses. Because these mechanisms rely heavily on government 145 regulation, negotiation, and price-setting, they have gained little favor in the U.S.

Pharmaceutical policy in the U.S. is not hampered by a lack of expert recommendations (13-15),

147 but rather by lack of political will to act upon them on a scale that could benefit the nation as a whole.

148 For example, the National Academies of Science, Engineering and Medicine recently outlined a series

149 of evidence-based recommendations to improve medication affordability (16). While the task force

150 provided actionable guidance to federal agencies who have legal authority to implement specific

151 recommendations, two factors act to reinforce the status quo: 1) the approach for reform remains

152 fragmented, and 2) the pharmaceutical market is an evolving and complex endeavor for which

153 continued and careful examination of evidence is needed to evaluate the success (and failure) of policy 154 recommendations.

More coherent and consistent pharmaceutical policies are needed at the national level to ensure

156 Americans have equitable access to affordable prescription medications. The ATS supports policies

157 that give people the best available resources to manage their health, including affordable access to

158 pharmaceutical therapies while also supporting innovation in the development of new efficacious

159 treatments. To meet these challenges, this ATS policy statement recommends the establishment of a

160 publicly funded, politically independent, impartial entity to systematically draft evidence-based

161 pharmaceutical policy recommendations at the national level. This statement outlines key features of

162 the pharmaceutical supply chain that impact people with chronic respiratory conditions, highlights

163 international examples of how those features can be better managed, and concludes with our rationale

164 of how such an entity can be integrated into our unique political system to provide impactful regulatory 165 guidance.

166 DESCRIPTION OF COMMITTEE COMPOSITION AND ACTIVITY

A multidisciplinary expert committee developed this policy statement. A full working group was recruited at the initiation of the project, comprising experts in health policy, pharmaceutical policy and pharmaco-economics, behavioral and translational science, pediatric and adult respiratory medicine, 
170 industry, and patients. Conflicts of interest were vetted and managed according to the policies and

171 procedures of the ATS. This policy statement was developed through an iterative, consensus-based

172 process that included face-to-face meetings, electronic polls and correspondence, and teleconferences.

173 The full committee participated in topic-focused writing committees, and provided expertise on various

174 sections of the statement. A writing committee consisting of the co-chairs drafted the initial policy

175 statement. The statement was reviewed by the full committee on multiple occasions and ultimately

176 approved by the ATS Board of Directors.

\section{THE PHARMACEUTICAL SUPPLY CHAIN IN THE UNITED STATES}

The current market for prescription medications is complex and contains numerous government and private entities that regulate, develop, distribute, dispense and purchase prescription medications. Recommendations addressing affordable access to pharmaceutical therapies must acknowledge the diverse roles that these entities play in making medications available to patients. We highlight the contributions of and structural challenges facing manufacturers, distributers, insurers, and prescribers as they seek to control growing pharmaceutical costs for patients with chronic lung disease.

\section{Manufacturers and Patent Laws}

Pharmaceutical manufacturers engage in drug research, development, evaluation, licensing and marketing with the aim of distributing therapies for a range of diseases. Pharmaceutical manufacturers are an important entity ensuring that innovative therapies are made available to the public. Although physician marketing and direct-to-consumer advertising are often a focus of scrutiny when it comes to examining costs, one of the most costly investments made by manufacturers includes the complex clinical trials required to evaluate efficacy, inform safety, and meet regulatory requirements (16).

A number of legal protections including regulatory and patent-related exclusivity prolong the lifespan of branded therapies, allowing patient cost of medications to be influenced by the wholesale price set by manufactures (14). While patent exclusivity is intended to reward novel drug development, it can 
also protect profits at the expense of lower prices for respiratory medicines, particularly those delivered by inhalation. For example, the U.S. FDA banned the production and sale of all chlorofluorocarbon (CFC)-based albuterol inhalers in 2008 in response to an international agreement to reduce environmental contaminants that were depleting the ozone layer. As an unintended consequence, generic CFC inhalers were replaced by more expensive, patented hydrofluoroalkane inhalers prompting higher OOP costs for patients, especially those privately insured (17). At this time, there are still no marketed generic albuterol inhalers. In addition to this unusual circumstance, respiratory medicines delivered by inhalation are more protected from generic competition than orally ingested medications because of the complexity of patent law involved in proprietary pump designs, delivery systems, formulations and production processes that hinder generic competition when the active ingredient may no longer be patent-protected. Oversight provided by a patient-oriented, politically independent, entity could help detect and mitigate unintended consequences of regulatory actions that sustain high prescription medication prices.

\section{Distribution Channels and Insurers}

Prescription medications are made available to patients only after passing through several intermediaries. Most recently, Pharmacy Benefit Managers (PBMs) have become influential intermediaries that secure prescription medications from manufacturers on behalf of health plans and employers. While pharmacies often provide patients with direct access to prescription medications, PBMs exist behind the scenes to develop and maintain formularies, sell medications and provide rebates to pharmacies, negotiate discounts and obtain medications from drug manufacturers, and process prescription drug claims on behalf of insurers. Three large PBMs serve $75 \%$ of the U.S. market (14). Although there is some consistency for public payers, like Medicaid, prices paid by private insurers are highly variable and typically lack price transparency. As a result, the prices paid insurers, pharmacies, and individual patients varies widely (18). Numerous intermediaries exist between manufacturers and patients; accordingly, each step in the supply chain is associated with mark-ups 
221 such that $\$ 1$ out of every $\$ 5$ spent on prescription drugs feeds profits in the distribution system (19). In

222 Europe, the price that such suppliers can charge is limited to a fixed percentage cost of the medication 223 being supplied.

Many insurance policies impose substantial cost-sharing requirements that impact OOP prescription drug spending including general medical deductibles, copayments and coinsurance. These requirements vary depending on whether the health insurance is publically or privately sponsored. For instance, high deductible private plans often require thousands of dollars in spending before subsidizing prescription medication costs. Alternatively, public plans, like Medicare, tend to impose smaller

229 deductibles but lack an annual expenditure cap exposing some beneficiaries (e.g., those undergoing cancer chemotherapy) to high OOP spending owing to co-insurance payments above the Medicare Part D catastrophic spending threshold. More routinely, Medicare patients with common chronic respiratory diseases like COPD may pay up to $\$ 1200$ annually to cover single maintenance inhaler treatment, a striking $79 \%$ of the total drug cost (20). Until the "donut hole" is phased out, those who use

$234>2$ inhalers will almost certainly reach this coverage gap where they are responsible for up to one-third of the drug costs until the catastrophic limit is reached, at which point they are responsible for $5 \%$. By federal regulation, Medicare patients cannot receive any copayment assistance from the pharmaceutical company marketing the product.

\section{Prescribers}

Pharmaceutical company relationships with physicians have long been scrutinized for their

242 have been enacted to reduce product detailing (22) in an attempt to reduce branded prescribing when 243 generic alternatives are available (23). An unintended consequence is the deceleration of the adoption 244 of new therapies (24). This is especially concerning for novel therapies for patients with rare diseases 245 where delayed uptake could deprive these patients of clinically beneficial therapies. 
Over time, more prescribers have come to recognize that treatment costs can impact adherence with treatment recommendations and/or place undue financial burden on patients (25-26). For many lung diseases, such as asthma and COPD, few suitable generics are available. Prescribing branded medications in these circumstances forces patients to pay more because these medications are typically placed in higher formulary tiers with larger copayments. Thus, prescribers are placed in a catch-22 where prescribing efficacious treatments may not offer the expected benefits owing to greater non-adherence as a result of cost.

Prescribers often lack adequate information or training to prescribe in a cost-conscious manner to improve affordability. Given large heterogeneity between health insurance formularies, costs for the same treatments are highly variable between patients. Formularies change frequently making it even more challenging for prescribers to address affordability for individual patients. Because hospitals have unique formularies, patients may be prescribed one type of medication prior to hospitalization, given a different one during the hospitalization, and then be prescribed that inhaler at discharge—meaning patients may, at times, be purchasing duplicative medications.

\section{PHARMACEUTICAL SUPPLY CHAIN IN THE INTERNATIONAL CONTEXT}

In an effort to ensure the safety, availability and access to health promoting pharmaceutical products worldwide, the World Health Organization (WHO) released evidence-based guideline to assist national policy-makers and other stakeholders to manage pharmaceutical prices (27).

Recommendations included greater regulation of mark-ups in the pharmaceutical supply chain, tax exemptions for pharmaceutical products, use of external reference pricing, promotion of generic medicines, and greater use of health technology assessments (27). These recommendations are widely used by high income countries, but not the US.

Outside of the US, the European Union (EU) comprises the second largest concentration of pharmaceutical industries and suppliers of patented medications. For over two decades, EU countries have been focusing on containing pharmaceutical expenditures and ensuring affordable access to 
272 medicines (29). Although, prescription medication prices are higher in the US than any other advanced

273 nation, prices in the EU have been rising (28) for many years as well (14). Several features of EU

274 countries help explain their lower prices. In general, the EU has a greater commitment to fulfill a right to 275 health care and essential medicines for its citizens (29). They also work to ensure that health policies in 276 member nations do not disrupt the goals and operation of the internal EU market-- a larger EU objective 277 (29). Thus EU pricing reflects that overarching goal. While individual countries have some leeway in 278 price setting, all member nations must comply with EU provisions governing transparency, regulation, 279 and approval. One common approach for individual EU countries is to use external reference pricing280 that is, setting their prices based on comparison prices in other member nations, often adopting the 281 lowest price. Canada has also adopted reference pricing using EU member nations as common 282 comparators. The European Commission, an elected group represented by each member country that 283 maintains the general interests of the EU and can propose legislation, has been examining many of the 284 same issues that face the U.S. in pharmaceutical policy reform. Examples of these issues include 285 adequate generic supply and the pharmaceutical lobby.

286 Many countries with universal healthcare and/or multi-payer systems have national organizations 287 responsible for conducting health technology assessments that generate evidence to support 288 reimbursement decisions and price setting, including Australia, Canada, Germany, Ireland, and the 289 United Kingdom. Organizations adopt a range of review criteria including clinical, comparative, and 290 cost-effectiveness evidence to drive decision-making. In the U.S., these same approaches have been 291 undertaken by non-governmental organizations such as the Institute for Clinical and Economic Review 292 and individual physician and patient advocacy groups; however, these efforts are isolated, underfunded 293 and their work is not widely used in practice.

\section{RECOMMENDATIONS}


Recommendation 1: The U.S. government should establish and financially support a politically independent, impartial expert advisory committee tasked with making evidence-based recommendations on pharmaceutical policy. Their overarching goal would be to ensure affordable access to life-preserving medications while retaining consumer choice, promoting future innovation, and respecting our market-based economy.

Without a single entity to coordinate and/or regulate the activities of the various parties comprising the pharmaceutical supply chain in the US, broad adoption of evidence-based policies has fallen behind that of other economically developed nations. Accordingly, patients are unduly burdened by high treatment costs, and as a result, experience greater morbidity due to cost-related non-adherence. Over the past decade, numerous entities have proposed policies to curb the rise in prescription medication prices including encouraging greater price transparency, changing patent law and adopting valuebased purchasing (16). However, lacking any clear standing, these recommendations have been largely ignored. The National Academies of Science, Engineering and Medicine recently recommended numerous, targeted, evidence-based reforms based on the best available evidence to address inefficiencies in the pharmaceutical supply chain (16). While our committee is supportive of many of these recommendations, we intentionally refrained from championing any particular strategy owing to our belief that adopting highly targeted policies in a piece-meal fashion will not sufficiently address the long-term challenges posed by a complex, ever-evolving system (16).

Instead, the problem calls for a permanent entity with sufficient standing to confront entrenched stakeholders and adapt to changing circumstances. To address this long-term need, we unanimously recommend that the U.S. government establish and financially support an independent expert advisory committee tasked with making recommendations on pharmaceutical policy. The fact that $75 \%$ of Americans already favor the creation of an independent group with specific focus on the costs of prescription medicines lends credence to the acceptability of our recommendation (30). 
One may view this recommendation as a "punt" by failing to make specific, targeted recommendation affecting obvious inefficiencies in the current pharmaceutical market. However, the committee concluded that there are two necessary requisites to realize pharmaceutical policy reform in the U.S: impartiality and capacity. Due to close financial relationships between industry and the medical profession it is impossible for organizations like the ATS to manage the actual or perceived conflicts of interest that exists between its members and industry. This makes it difficult for professional societies to make independent and objective recommendations with suitable traction to bring about meaningful change. Second, individual professional societies lack the capacity to shoulder the long-term burden needed to reform pharmaceutical policy. While such societies should inform and influence policy, their voice alone is insufficient to realize the substantial changes that are needed.

Given the magnitude of the challenge, only the federal government is positioned to facilitate, fund and organize this effort. However, public distrust in government and hyper-partisanship would invariably threaten the impartiality of such an entity without a high degree of independence from political forces. Therefore, we propose the creation of an independent entity for pharmaceutical reform be modeled after the successful example of the United States Preventive Services Task Force (USPSTF). This entity, supported by the Agency for Healthcare Research and Quality, has served in an independent, impartial advisory capacity since 1984 (31).

The example of the USPSTF demonstrates how the federal government can effectively advance health policy formulation independent of partisan influence. The USPSTF is comprised of an independent group of national experts in prevention and evidence-based medicine that works to improve the health of all Americans by making evidence-based recommendations about clinical preventive services. Because its recommendations are not subject to government approval, they are highly respected by the general public, public officials, health care entities and clinicians. A notable example of how the USPSTF has exerted tangible impact on national health policy is reflected by the Affordable Care Act requirement that all public and private insurers must provide recommended preventive services without patient cost-sharing. Admittedly, this provision only had incremental impact 
on our health system, but as recently pointed out, "incremental change is not necessarily inconsequential change" (32).

Other similar organizations exist that operate independently in order to support evidence-based practice and policy, and their operating procedures could be considered as models for the proposed entity, including the Medicare Payment Advisory Commission (MedPAC), and the Congressional Budget Office.

\section{Structure of an independent expert advisory committee}

An independent, expert advisory committee would be comprised of national pharmaceutical policy experts representing a broad range of stakeholders including clinicians, pharmacists, researchers, public health practitioners, patient advocates, industry and legal experts, and economists. Like the USPSTF, individuals would be publicly nominated. Members would be appointed to serve fixed terms, and screened to ensure that they have no substantial conflicts of interest that could impair the scientific integrity of the entity's work. Similar to MedPAC, members would ultimately be confirmed by the Comptroller General. A clear set of conflict of interest protocols would be established to monitor and manage conflicts.

The newly proposed entity would be tasked with making evidence-based recommendations regarding the myriad of factors that influence access to affordable prescription medications. Their overarching goals would be to make recommendations that if implemented, would likely result in improved access to life-preserving medications, while retaining consumer choice, promoting future innovation and respecting our market-based economy. The entity would require an appropriate infrastructure to conduct evidence reviews. They would necessarily seek feedback from professional societies, advocacy groups, and expert consultants as needed. This would provide an important avenue for ATS to shape important policy recommendations that impact patient affordability of respiratory therapies. Recommendations would contain detailed information about the primary evidence behind it, and how to implement the recommendation. Like the USPSTF, when there is not enough evidence to support a recommendation, the entity would make a clear call for needed research. The 
373 entity would have at least two annual required reports to the U.S. Congress, who along with

374 government agencies with legislative authority, would ultimately enforce and implement

375 recommendations. Reports would make clear recommendations to government agencies (e.g FDA)

376 with definitive legislative authority or to the U.S. Congress to grant legislative authority where necessary 377 (16). medicines more affordable (16), and others (14-15) have identified several key areas for further examination to inform evidence-based pharmaceutical policy reform that this new entity can draw from to initiate their scope of work- including value-based pricing, transparency, and uniform negotiation. Table 1 highlights priorities in pharmaceutical policy reform in the U.S. to support the ATS mission and patient affordability and access.

Recommendation 2: An immediate priority of the aforementioned committee is to examine the evidence and make recommendations to have essential medicines with established clinical benefit from each drug class in all Tier 1 formularies, and to reduce barriers to timely generic drug availability.

A critical issue for patients with respiratory disease are the high out-of-pocket costs of inhalers owing to lack of low-cost generic availability and variation in coverage on insurance formularies. Advair for example, a commonly prescribed medicine for asthma and COPD, had its U.S. patent expire in 3912010 , but a generic version has yet to enter the market at the time of this writing. As previously mentioned, generic availability of Albuterol has also faced challenges. If the proposed entity had been around at the time generic CFC inhalers were replaced by patented, hydrofluoroalkane inhalers, they would have been equipped to anticipate the lack of availability of albuterol generics as a result of the change and the anticipated cost consequences. Their work could have therefore addressed the issue and perhaps avoided the untenable situation we are in now. Despite the established clinical benefit of 
not guaranteed on Tier 1 formularies of all insurance plans where out-of-pocket costs would be the lowest for patients compared to other tiers. Although the ACA established an essential benefits list that includes prescriptions, there are not specific protections for certain medicines, or specific guidance or incentives around formulary tiering. A re-examination of essential prescription lists are needed with more uniformity in how insurance plans cover medicines from each drug class at minimal cost to patients.

Entry of generic medicines into the market has historically shown that the price of previously patented medicines can drop substantially as a result of increased competition among manufacturers. However, delays in generic entry of medicines and the rising costs of generics in general due to fewer suppliers have created a situation that requires intervention. Key issues that an established entity would provide evidence review and recommendations on would include industry practices to extend patent exclusivity, FDA backlogs of safe generic entry onto the market, and increasing availability of manufactures and their ability to compete (16).

\section{CONCLUSION}

The ATS supports policies that improve the health and well-being of patients with chronic lung disease. At present, pharmaceutical costs of common therapies are burdensome for many patients and a major barrier to medication adherence. As other countries have demonstrated, this burden can be mitigated. We recommend the federal government establish and fund an independent, impartial entity to spearhead U.S. pharmaceutical policy reform and prioritize the availability of low-cost generic medicines with proven clinical benefit. This entity would shape pharmaceutical policy reform by developing evidence-based policies that are congruent with international best practices yet consistent with our unique national values. We believe that an independent, impartial entity dedicated to tackling the complex issues involving the development, manufacture, distribution, and sale of pharmaceutical products would be best-positioned to improve our patients' access to affordable, life-preserving and lifeenhancing therapies long-term. Such an entity represents a best first step for incremental, yet meaningful, reform of U.S. pharmaceutical policy. 
424 Members of the Committee:

425 Minal R. Patel, PhD, MPH (Co-Chair)

426 Joe K. Gerald, MD, PhD (Co-Chair)

427 Lynn B. Gerald, PhD. MSPH (Co-Chair)

428 Valerie G. Press, MD, MPH (Co-Chair)

429 Teresa Barnes, BA

430 Kathryn Blake, Pharm.D.

431 Lee K. Brown, MD

432 Richard Costello, MD

433 Courtney Crim, MD

434 Gary Ewart, MHS

435 Mark Forshag, MD, MHA

436 Andrea Gershon, MD, MSc, FRCPC

437 Christopher H. Goss, MD, MS, FCCP

438 MeiLan K. Han, MD, MS

439 Todd A. Lee, PhD, PharmD

440 Nuala Moore, MA

441 Stuart Sweet, MD, PhD

442

443 
446 1. Centers for Disease Control and Prevention. Chronic Disease Prevention and Health Promotion, 2018. Available from: https://www.cdc.gov/chronicdisease/index.htm.

2. Kantor ED, Rehm CD, Haas JS, Chan AT, Giovannucci EL. Trends in Prescription Drug Use Among Adults in the United States From 1999-2012. JAMA. 2015;314(17):1818-1831. doi:10.1001/jama.2015.13766.

451 3. Dieleman JL, Baral R, Birger M, et al. US Spending on Personal Health Care and Public Health, 452 1996-2013. JAMA. 2016;316(24):2627-2646. doi:10.1001/jama.2016.16885.

453 4. Health Care Cost Institute. Out-of-pocket spending trends (2013). Issue Brief \#9, October 2014. $454 \quad$ Washington, DC: Health Care Cost Institute.

455 5. Cohen RA, Kirzinger WK. Financial burden of medical care: a family perspective. NCHS Data Brief. $456 \quad 2014 ; 142: 1-8$.

457 6. Paez, Kathyrn Anne. Rising Out-of-Pocket Spending for Chronic Conditions: A Ten Year Trend. $458 \quad$ Health Affairs. 2009; 28(1):15-25. doi:10.1377/hlthaff.28.1.15.

459 7. McHorney CA, Victor Spain C, Alexander CM, Simmons J. Validity of the adherence estimator in 460 the prediction of 9-month persistence with medications prescribed for chronic diseases: a 461 prospective analysis of data from pharmacy claims. Clin Ther. 2009;31(11):2584-607. 462 doi:10.1016/j.clinthera.2009.11.030.

463 8. Heisler M, Choi H, Rosen AB, Vijan S, Kabeto M, Langa KM, et al. Hospitalizations and deaths 464 among adults with cardiovascular disease who underuse medications because of cost: a 465 longitudinal analysis. Med Care. 2010;48(2):87-94. doi: 10.1097/MLR.0b013e3181c12e53. underuse, and outcomes among diabetes patients in three systems of care. Med Care. 2004;42(2):102-9. 
10. Sarnak, Dana O. Paying for Prescription Drugs Around the World: Why Is the US and Outlier? The Commonwealth Fund. October 2017.

11. Patel MR, Piette JD, Resnicow K, Dobson-Kowalski T, Heisler, M. Social determinants of health, cost-related non-adherence, and cost-reducing behaviors among adults with diabetes: findings from the National Health Interview Survey. Med Care. 2016;54(8):796-803.

12. Organisation for Economic Co-operation and Development. (2015). Fiscal Sustainability of Health Systems. OECD Publishing, Paris.

13. Hwang TJ, Kesselheim AS, Sarpatwari A. Value-Based Pricing and State Reform of Prescription Drug Costs. JAMA. 2017;318(7):609-610. doi:10.1001/jama.2017.8255.

14. Kesselheim AS, Avorn J, Sarpatwari A. The High Cost of Prescription Drugs in the United States: Origins and Prospects for Reform. JAMA. 2016;316(8):858-871. doi:10.1001/jama.2016.11237.

15. Daniel H; Health and Public Policy Committee of the American College of Physicians. Stemming the Escalating Cost of Prescription Drugs: A Position Paper of the American College of Physicians. Ann Intern Med. 2016;165(1):50-52. Doi: 10.7326/M15-2768.

16. National Academies of Sciences, Engineering, and Medicine. 2017. Making Medicines Affordable: A National Imperative. Washington, DC: The National Academies Press.

17. Jena AB, Ho O, Goldman DP, Karaca-Mandic P. The Impact of the US Food and Drug Administration Chlorofluorocarbon Ban on Out-of-pocket Costs and Use of Albuterol Inhalers Among Individuals With Asthma. JAMA Intern Med. 2015;175(7):1171-9. 10.1001/jamainternmed.2015.1665.

18. Kullgren JT, Segel JE, Peterson TA, Fendrick AM, Singh S. Availability and variation of publicly reported prescription drug prices. Am J Manag Care. 2017;23(7):444-448.

19. Sood N, Shih T, Van Nuys K, Goldman D. (2017). The flow of money through the pharmaceutical distribution system. Accessed from: http://healthpolicy.usc.edu/documents/USC\%20Schaeffer Flow\%20of\%20Money 2017.pdf 
20. Tseng CW, Yazdany J, Dudley RA, DeJong C, Kazi DS, Chen R, Lin GA. Medicare Part D Plans' Coverage and Cost-Sharing for Acute Rescue and Preventive Inhalers for Chronic Obstructive Pulmonary Disease. JAMA Intern Med. 2017;177(4):585-588. doi:10.1001/jamainternmed.2016.9386.

21. Ornstein C, RG Jones RG, Tigas M. Dollars for Doctors. New York, NY: ProPublica, 2016.

22. Larkin I, Ang D, Steinhart J, Chao M, Patterson M, Sah S, Wu T, Schoenbaum M, Hutchins D, Brennan T, Loewenstein G. Association Between Academic Medical Center Pharmaceutical Detailing Policies and Physician Prescribing. JAMA. 2017;317(17):1785-1795. doi:10.1001/jama.2017.4039.

23. Larkin I, Ang D, Avorn J, Kesselheim AS. Restrictions on pharmaceutical detailing reduced offlabel prescribing of antidepressants and antipsychotics in children. Health Aff (Millwood). 2014;33(6):1014-1023. doi: 10.1377/hlthaff.2013.0939.

24. DeJong C, Dudley RA. Reconsidering Physician-Pharmaceutical Industry Relationships. JAMA. 2017;317(17):1772-1773.

25. Ubel PA, Jagsi R. Promoting population health through financial stewardship. N Engl J Med. 2014;370(14):1280-1. doi: 10.1056/NEJMp1401335.

26. Ubel PA, Abernethy AP, Zafar SY. Full disclosure--out-of-pocket costs as side effects. N Engl J Med. 2013;369(16):1484-6. doi: 10.1056/NEJMp1306826.

27. World Health Organization. WHO Guideline on Country Pharmaceutical Pricing Policies. (Geneva); 2013.

28. Wouters OJ, Kanavos PG. A comparison of generic drug prices in seven European countries: a methodological analysis. BMC Health Services Research. 2017;17:242. doi:10.1186/s12913-0172184-5.

29. European Union. Official Website of the European Union, 2018. Accessed from: https://europa.eu/european-union/index en 
519 30. Kaiser Family Foundation. Public opinion on prescription drugs and their prices. 2017. Accessed 520 from: https://www.kff.org/slideshow/public-opinion-on-prescription-drugs-and-their-prices/

521 31. US Preventive Services Task Force. About the USPSTF. 2018. Accessed from:

$522 \quad$ https://www.uspreventiveservicestaskforce.org/Page/Name/about-the-uspstf.

523 32. Frakt $A B$ and Chernew ME. The Importance of Relative Prices in Health Care Spending. JAMA.

$524 \quad$ 2018; 319(5):441-442. doi:10.1001/jama.2017.20047.

525 
Table 1. Priorities in pharmaceutical policy reform in the U.S. to support the ATS mission and patient affordability and access ${ }^{16,14}$

Expand flexibility of formulary design to mitigate cost burdens to patients. Essential respiratory medicines with established clinical benefit should be available in Tier 1 formularies

Reduce barriers to timely generic drug availability and foster competition to ensure continued affordability

Promote expeditious market entry of international providers of generic and novel medicines.

Establish uniform and meaningful negotiation with producers and suppliers of medicines in the pharmaceutical supply chain

Establish price transparency of financial flows through the pharmaceutical supply chain

Establish a cost-effectiveness research agenda and action plan that develops value metrics for therapies and a uniform pharmaceutical value framework

Expand value-based pricing and benefit design, including demonstration projects to test alternative payment models on outcomes and costs

Incentivize development of truly new medicines

Ensure that financial incentives for the prevention and treatment of rare diseases are not extended to widely sold drugs

Expand stock albuterol programs in schools within all 50 states

Establish an infrastructure for ongoing examination of patient affordability, and availability and access to assistance programs

Define a list of essential medicines and routinely examine this list

Establish policies to reduce academic detailing, lobbying, direct-to-consumer drug advertising, and anticompetitive deals

Examine expenses and processes between $\mathrm{NIH}$ and small-industry supported early stage drug development research and that of large pharmaceutical manufacturers

Modify requirements for the award and extension of exclusivity rights 